\title{
Development and validation of a computer program using Bayes's theorem to support diagnosis of rheumatic disorders
}

\author{
H J Bernelot Moens, J K van der Korst
}

\begin{abstract}
A computer program has been developed to support the differential diagnosis of major rheumatic disease categories. Knowledge for the program was derived from the sensitivity and specificity of findings in a group of $\mathbf{1 0 5 2}$ consecutive patients of a rheumatological outpatient clinic. Computer predictions based on two variations of Bayes's theorem were compared with 'gold standard' diagnoses in $\mathbf{5 7 0}$ test cases from the same clinic. The results demonstrate the feasibility of this approach, but also that refinements of both the method of calculation and the knowledge are required.
\end{abstract}

Many rheumatological diagnoses are based on a combination of signs, symptoms, or tests rather than on a single pathognomonic feature. This has motivated the development of criteria for the diagnosis and classification of rheumatic diseases. Many of these criteria have been helpful both in gaining insight into pathogenesis and in improving treatment, and are now widely accepted. A related characteristic of diagnoses of rheumatic syndromes is uncertainty, to which three factors contribute. Firstly, the development from early signs to well defined syndromes is often slow. Secondly, syndromes often overlap. Thirdly, diagnoses may be altered during the course of a disease-for example, when a typical presentation of polymyalgia rheumatica develops into classical rheumatoid arthritis. Unfortunately, few existing criteria sets reflect the uncertainty or probability of diagnoses. In the case of rheumatoid arthritis the terms probable and definite or classical disease were actually abandoned in the 1987 criteria. $^{1}$ The inherent uncertainty and the reliance on combinations of findings make the diagnostic process in rheumatology an interesting area for the application of methods from the field of artificial intelligence. In medicine this scientific discipline is often referred to as computer assisted diagnosis or computer assisted medical decision making. ${ }^{23}$ The practical result is called an 'expert system': a computer program that simulates the problem solving behaviour of a human expert. To date four prototypes of rheumatological expert systems have been described. ${ }^{4-9}$ These made use of criteria tables, rules, or fuzzy set theory to produce diagnoses at different levels of confidence.

Here we report another effort. Our primary goal was to calculate the probability of important rheumatic disease states or categories by applying Bayes's rule or 'theorem' to findings from a patient's medical history, physical examinations, and diagnostic tests. We describe here the design of the computer program, our approach to obtaining the information required for the application of Bayes's theorem, and the results of a first evaluation in a test set of patient data.

\section{Patients and methods \\ PATIENTS}

From September 1987 to September 1988 data were collected on consecutive patients who had been newly referred to the rheumatological outpatient clinic. During the first examination rheumatologists recorded 95 clinical findings on a form that had been provided for the purposes of this study. The results of radiography, blood and other tests were obtained from the patients' charts after the patients' first visits. Forms were received for 1588 patients. The notes for 1570 of these patients were available for review.

The stock examples that are used as references or types in designing and testing an expert system are referred to as the 'gold standard' for that system. To establish a gold standard diagnosis for each case two aspects of diagnosis were taken into consideration: outcome over time and consensus among rheumatologists. This was effectuated by a review of the diagnoses made by the rheumatologist treating the patient by a second rheumatologist (HJBM), six to 12 months after the first visit. Whenever there was disagreement the opinion of a third rheumatologist was asked. Clinical diagnoses were made in 15 categories and at two levels of confidence (table 1). A diagnosis was marked as 'definite' when it was made at that level by two rheumatologists. If there was partial agreement, or if

Table 1 Clinical 'gold standard' diagnoses in 1570 consecutive outpatients. More than one diagnosis was made in $602(38 \%)$ cases. Results are given as number (percentage) of patients

\begin{tabular}{lrr}
\hline Diagnostic category & Definite & Possible \\
\hline Rheumatoid arthritis & $93(5 \cdot 9)$ & $95(6 \cdot 1)$ \\
Juvenile rheumatoid arthritis & $17(1 \cdot 1)$ & $8(0 \cdot 5)$ \\
Systemic autoimmune disease & $10(0 \cdot 6)$ & $56(3 \cdot 6)$ \\
Reactive arthritis/Reiter's disease & $11(0 \cdot 7)$ & $19(1 \cdot 2)$ \\
Ankylosing spondylitis & $35(2 \cdot 2)$ & $15(1 \cdot 0)$ \\
Psoriatic arthritis & $27(1 \cdot 7)$ & $16(1 \cdot 0)$ \\
Bacterial arthritis & $0(0 \cdot 0)$ & $4(0 \cdot 3)$ \\
Polymyalgia rheumatica & $8(0 \cdot 5)$ & $12(0 \cdot 8)$ \\
Gout or pseudogout & $18(1 \cdot 1)$ & $41(2 \cdot 6)$ \\
Non-specific arthritis & $191(12 \cdot 2)$ & $3(0 \cdot 2)$ \\
Osteoarthrosis & $318(20 \cdot 3)$ & $127(8 \cdot 1)$ \\
Periarthritis humeroscapularis & $141(9 \cdot 0)$ & - \\
Tendinitis or bursitis & $94(6 \cdot 0)$ & - \\
Other disease & $90(5 \cdot 7)$ & $7(0 \cdot 4)$ \\
Non-specific pain $\dagger$ & $799(50 \cdot 9)$ & - \\
\hline
\end{tabular}

"Patients with musculoskeletal complaints caused by diseases such as sarcoidosis, malignancy, thyroid dysfunction, carpa tunnel syndrome, etc.

tPatients with musculoskeletal complaints (primarily low back pain or joint pains, or both) that could not be classified otherwise. 
doctors agreed that the level of confidence was less than definite, the diagnosis was recorded as 'possible'. To ensure classification of all patients the categories 'other disease' and 'no diagnosis' were introduced. The latter applied mainly to patients in whom no physical or other abnormality could be shown and who were consequently given descriptive diagnoses such as non-specific joint pain or low back pain. As bacterial arthritis is rarely encountered in the outpatient clinic we also recorded retrospective data from the notes of 52 previously studied patients. ${ }^{10}$ More than one diagnosis per case was allowed. For example, a gold standard diagnosis might be: degenerative joint disease (definite), gout (possible), bacterial arthritis (possible).

\section{DEVELOPMENT OF A COMPUTER PROGRAM}

A computer program was developed to predict the probability of the diagnoses from a set of patient findings. Bayes's rule was implemented to calculate the probabilities. Diagnostic outcomes were linked with descriptive information to provide advice on management and treatment and to enhance the system's educational value. Practical situations impose time constraints. As the system is also intended for use in patient care it was necessary to enable the user to enter data and obtain results within one minute.

\section{COMPUTATION OF DIAGNOSTIC PROBABILITIES}

Bayes's theorem describes the relation between prior probability, sensitivity, and specificity, and posterior probability. ${ }^{2} 11$ Prior and posterior probabilities are also known as pre-test and post-test probabilities. Multivariate versions of Bayes's rule require that findings be conditionally independent. A matrix representing the strength of association between individual findings was used in conjunction with clinical experience to make an initial selection of the 95 findings on the patient data form.

Two variations of Bayes's equation were compared. The first represents the classical bayesian approach with the formula:

$p\left(D_{i} \mid s_{1}, \ldots, s_{n}\right) \alpha p\left(s_{1} \mid D_{i}\right) \times \ldots \times p\left(s_{n} \mid D_{i}\right) \times p\left(D_{i}\right)$

Here, $p\left(D_{i}\right)$ is the prior probability of the ith disease and $p\left(s \mid D_{i}\right)$ is the probability of signs being present in the ith disease. For computational reasons the program uses the natural logarithms of the sensitivities. These are summed and converted to obtain the odds for each disease. The probabilities are then normalised to add to 1 or $100 \%,{ }^{*}$ which is indicated by the sign of proportionality.

The second method is a modification described by Spiegelhalter. ${ }^{12} \mathrm{~A}$ modified likelihood ratio or weight, $w$, is calculated for each answer or symptom (s) as:

$$
w(s \mid D)=\log _{e} \frac{\left(r_{D}+0.5\right) /\left(n_{D}+0.5\right)}{\left(r_{\bar{D}}+0.5\right) /\left(n_{\bar{D}}+0.5\right)}
$$

where $\mathbf{n}$ is the number of cases with the disease (D) or without $(\overline{\mathrm{D}})$ a particular diagnostic outcome, and $r$ is the number of patients with a particular finding in these groups. To compensate for dependence the weights were multiplied by $0 \cdot 8 .{ }^{12}$ The odds for each diagnosis are based on:

$\log _{e} \frac{p\left(D \mid s_{1}, \ldots, s_{n}\right)}{p\left(\bar{D} \mid s_{1}, \ldots, s_{n}\right)}=w\left(s_{1} \mid D\right)+\ldots+w\left(s_{n} \mid D\right)+\log _{e} \frac{p(D)}{p(\bar{D})}$

Odds were calculated by adding the weights of all answers to the logarithm of the odds for each disease. The exponents of the sum were then normalised.

In both models the prevalence of the diagnoses in the first 1000 patients was used as prior likelihood $\mathrm{p}(\mathrm{D})$.

\section{EVALUATION}

To verify the accuracy of the computer predictions a separate computer routine was created to diagnose a sequence of cases and compare the results with the consensus diagnoses that were used as gold standard. Parameters used to measure the performance were:

(1) Percentage of gold standard diagnoses correctly included among the five diagnoses with the highest predicted probabilities.

(2) Sensitivity and specificity for gold standard diagnoses, defined as the percentage of definite and possible doctor's diagnoses with a predicted probability of more than or less than 0.2 . The effect of variation of this threshold is illustrated by a receiver operating characteristic. ${ }^{11}$

(3) Average predicted probability by diagnosis, reported separately for definite and possible gold standard diagnoses and for diagnoses not made by the doctors.

(4) Average predicted probability by rank position and by level of probability. This answers the question whether a predicted probability of, for example, 0.8 corresponds to $80 \%$ of these cases in the test group having the predicted diagnosis. For each category the expected frequency of correct diagnoses is the number of cases multiplied by the average probability. The observed frequency is the actual fraction of cases in which the diagnosis was correct. Calculation of the standard error and the $\mathrm{Z}$ statistic were described by Spiegelhalter. ${ }^{12}$

(5) To allow comparison of different modifications of a model the use of an accuracy coefficient $Q$ has been proposed. ${ }^{2} 1314 \mathrm{We}$ further modified this coefficient to represent the average deviation from the probability of the gold standard. A perfect prediction for a definite gold standard diagnosis is assigned the value 1 ; a possible gold standard diagnosis, 0.5 ; and a diagnosis not included in the gold standard, 0 . The modified coefficient $Q^{\prime \prime}$ was calculated as:

$$
Q^{\prime \prime}=1-\frac{\sum\left|d_{i}-p_{i}\right|}{n}
$$

where $d_{i}$ is the assigned probability of the gold standard, $p_{i}$ is the probability predicted by the model for the ith prediction made by the computer, and $\mathbf{n}$ is the number of predictions given by the computer. In our model there were 15 predictions for each case. $Q^{\prime \prime}$ then can vary from 0 to 1 , where 1 is the best possible result. 
Results

THE COMPUTER APPLICATION*

The program that was developed runs under MS-DOS. Findings can be entered as answers to one or more of 74 multiple choice questions on medical history (32), physical examination (25), blood tests (seven), and radiography results (10). Answering 30 questions takes 60 seconds. Help with the definitions of findings is available to the user as the data are being entered. The speed of calculation of the probability of the 15 diagnoses in the current model is dependent on the number of questions answered and the type of processor. For 30 questions the time varies from 9.5 seconds on an 'XT' compatible microcomputer to less than three seconds on faster machines.

The conclusion is presented as a list of maximally five diagnoses ranked by probability. Given this information the user has options to: (a) calculate minimal and maximal posterior probabilities that would result from diagnostic tests; (b) search for further symptoms that would support the diagnoses listed; (c) consult descriptive information concerning one of the diagnoses. The descriptive information on findings and diagnoses is presented as pages of text, never exceeding 20 lines or one screen. The user may also consult these texts-for example, a dosage regimen, by entering a page number or free text, such as the name of a disease or drug. Each page contains a list of topics allowing the user to turn to another page with related information. This option is also used to incorporate a diagnostic algorithm as a separate form of decision support, which guides the user by question and answer to pages concerning specific diagnoses.

\section{KNOWLEDGE ACQUISITION}

Most patients in this study presented with relatively straightforward problems that could easily be classified by rheumatologists. In less than $5 \%$ of the cases the reviewing rheumatologist disagreed with the conclusion made by the doctor who treated the patient. The disagreement mainly concerned the estimated likelihood and in these cases the opinion of a third rheumatologist directed the classification of the gold standard diagnosis. Table 1 presents the

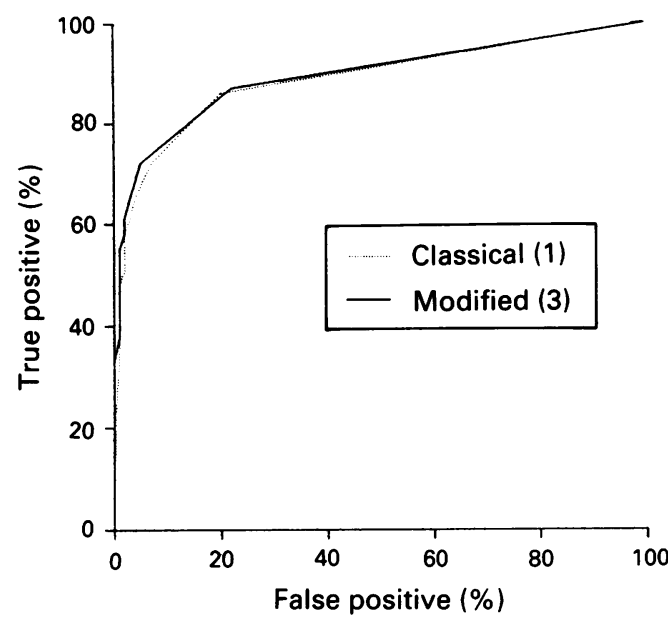

Receiver operating characteristics for diagnoses by two variations of Bayes's theorem in 570 test cases. True positive = percentage of computer diagnoses in agreement with a definite or possible gold standard diagnosis; false positive = percentage of computer diagnoses not in agreement with the gold standard diagnosis.

prevalence of gold standard diagnoses. The average age of the patients was 49 years (range $2-93)$. The ratio of women/men was $1061 / 509$ $(68 \% / 32 \%)$.

The patients were divided into two groups: a learning group, consisting of the 52 patients with bacterial arthritis studied retrospectively and the first 1000 patients studied prospectively, and a test group, consisting of the remaining 570 patients studied prospectively, to be used in evaluating the model. The data from the 1052 cases in the learning group were used to obtain the sensitivity and specificity of each finding (sign, symptom, or diagnostic test) for each diagnosis. With these data a matrix was constructed, in which the logarithms of the likelihood ratios for the classical method and a weight for each answer and each diagnosis for the modified method were tabulated.

\section{EVALUATION}

Computer predictions were made for each of the 570 cases in the test group. In this group doctors made 839 diagnoses, 694 definite and 145 possible. Of these, the classical model

Table 2 Average probability by diagnosis in 570 test cases for two variations of Bayes's formula

\begin{tabular}{|c|c|c|c|c|c|c|c|}
\hline \multirow[t]{2}{*}{ Disease category } & \multirow{2}{*}{$\begin{array}{l}\text { Number } \\
\text { of cases }\end{array}$} & \multicolumn{3}{|c|}{ Classical formula (1) } & \multicolumn{3}{|c|}{ Modified formula (3) } \\
\hline & & Definite $^{*}$ & Possible & Absent $^{*}$ & Definite & Possible & Absent \\
\hline Rheumatoid arthritis & 79 & 0.89 & 0.59 & 0.044 & 0.92 & 0.65 & $0 \cdot 050$ \\
\hline Juvenile rheumatoid arthritis & 14 & $0 \cdot 13$ & 0.09 & 0.001 & $0 \cdot 19$ & $0 \cdot 27$ & 0.004 \\
\hline Systemic autoimmune disease & 26 & $0 \cdot 12$ & $0 \cdot 20$ & 0.031 & 0.03 & $0 \cdot 17$ & 0.019 \\
\hline Reactive arthritis & 8 & 0.54 & 0.44 & 0.011 & 0.45 & 0.29 & 0.011 \\
\hline Ankylosing spondylitis & 21 & 0.83 & $0: 42$ & 0.007 & 0.87 & 0.56 & 0.060 \\
\hline Psoriatic arthritis & 10 & 0.67 & 0.01 & 0.004 & $0 \cdot 70$ & 0.02 & 0.006 \\
\hline Bacterial arthritis & $\mathbf{0}$ & - & - & 0.002 & - & - & 0.008 \\
\hline Polymyalgia rheumatica & 9 & 0.95 & 0.23 & 0.004 & 0.95 & $0 \cdot 17$ & 0.005 \\
\hline Gout or pseudogout & 22 & $0 \cdot 88$ & 0.41 & 0.009 & 0.94 & 0.43 & 0.013 \\
\hline Non-specific arthritis & 89 & $0 \cdot 31$ & 0.43 & 0.062 & $0 \cdot 26$ & $0 \cdot 11$ & 0.038 \\
\hline Osteoarthrosis & 157 & 0.54 & $0 \cdot 18$ & 0.051 & 0.59 & $0 \cdot 13$ & $0 \cdot 040$ \\
\hline Periarthritis humeroscapularis & 38 & 0.57 & - & 0.023 & 0.53 & - & 0.021 \\
\hline Soft tissue rheumatism & 28 & $0 \cdot 17$ & - & 0.033 & $0 \cdot 10$ & - & $0 \cdot 016$ \\
\hline Other disease & 33 & 0.04 & 0.09 & $0 \cdot 030$ & 0.03 & 0.07 & $0 \cdot 016$ \\
\hline Non-specific pain & 303 & 0.52 & - & 0.083 & 0.61 & - & 0.095 \\
\hline All diagnoses & 839 & 0.49 & 0.34 & 0.023 & 0.53 & $0 \cdot 34$ & 0.020 \\
\hline
\end{tabular}


Table 3 Performance by ranking of diagnoses in 570 test cases

\begin{tabular}{|c|c|c|c|c|c|c|c|c|c|c|}
\hline \multirow[t]{2}{*}{ Rank } & \multicolumn{5}{|c|}{ Classical formula (l) } & \multicolumn{5}{|c|}{ Modified formula (3) } \\
\hline & $P^{*}$ & $\operatorname{Exp}^{*}$ & $O b s^{*}$ & $S E^{*}$ & $Z^{*}$ & $P$ & $\operatorname{Exp}$ & Obs & $S E$ & $Z$ \\
\hline $\begin{array}{l}1 \\
2 \\
3 \\
4 \\
5\end{array}$ & $\begin{array}{l}0.76 \\
0.15 \\
0.05 \\
0.02 \\
0.01\end{array}$ & $\begin{array}{r}435 \\
84 \\
29 \\
12 \\
5\end{array}$ & $\begin{array}{r}427 \\
172 \\
73 \\
43 \\
20\end{array}$ & $\begin{array}{r}10 \cdot 2 \\
8 \cdot 5 \\
5 \cdot 2 \\
3 \cdot 4 \\
2 \cdot 3\end{array}$ & $\begin{array}{r}-0.8 \\
10.4 \\
8.5 \\
9.0 \\
6.4\end{array}$ & $\begin{array}{l}0.80 \\
0.11 \\
0.04 \\
0.02 \\
0.01\end{array}$ & $\begin{array}{r}456 \\
63 \\
22 \\
12 \\
7\end{array}$ & $\begin{array}{r}449 \\
156 \\
75 \\
29 \\
18\end{array}$ & $\begin{array}{l}9 \cdot 5 \\
7.5 \\
4.6 \\
3.4 \\
2.5\end{array}$ & $\begin{array}{r}-0.8 \\
12.5 \\
11.4 \\
5.0 \\
4.5\end{array}$ \\
\hline
\end{tabular}

*Average predicted probability; Exp=expected number of correct diagnoses (570 $\times$ average probability); Obs=observed number of cases in each category with a correct diagnosis

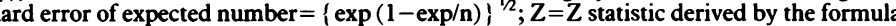
$Z=$ (observed-expected)/(SE). Values of $Z$ between 2 and -2 are optimal (see ref 12).

ranked $735(88 \%)$ and the modified model 727 $(87 \%)$ among the first five diagnoses. When only diagnoses with a predicted probability greater than $0 \cdot 20$ were counted, sensitivity was $62 \%$ for both formulas, while specificity was $97 \%$ and $98 \%$ respectively for the classical formula (1) and the modified formula (3). The result of variation of the threshold between 0 and 1 is illustrated by the receiver operating characteristic curve (figure). The average probability by diagnosis (table 2 ) shows considerable differences across diagnostic categories. Accuracy is high for diseases with unambiguous characteristics such as rheumatoid arthritis and ankylosing spondylitis, but inadequate for the broad categories systemic autoimmune disease, soft tissue rheumatism, and other disease. The relatively poor performance with respect to juvenile rheumatoid arthritis is because of confusion with rheumatoid arthritis, owing partly to the omission of age at onset as a separate finding. The modified 'weights' formula gave rise to a higher overall probability for definite diagnoses and lower probabilities for diagnoses not made by any doctors. It therefore produced slightly better results than the classical formula (1). Evaluation by rank order shows that the first diagnosis presented by the program was correct in $435(76 \%)$ and $456(80 \%)$ cases respectively (table 3 ). The average probability assigned to the first diagnosis was 0.76 and 0.80 respectively, which suggests high accuracy of the predicted probability of the first diagnosis. Evaluation by prediction level further illustrates the relation between expected and observed numbers of correct diagnoses (table 4).

Overconfidence, corresponding to negative $Z$ values, occurred mainly with probabilities above 0.95 , while computer estimates of probabilities below 0.20 were too low, which resulted in positive $Z$ values. Table 5 gives the
Table 5 Modified accuracy coefficient $Q^{\prime \prime}$

\begin{tabular}{lcll}
\hline Diagnosis & $n^{*}$ & $\begin{array}{l}\text { Classical } \\
\text { formula }(1)\end{array}$ & $\begin{array}{l}\text { Modified } \\
\text { formula (3) }\end{array}$ \\
\hline Definite & 694 & 0.489 & 0.526 \\
Possible & 145 & 0.616 & 0.600 \\
Not made & 7711 & 0.977 & 0.980 \\
All & 8550 & 0.931 & 0.937 \\
\hline
\end{tabular}

${ }^{*} n=$ number of predictions made by the model; $Q$ ": see 'Evaluation' section for explanation: optimal value is 1 , worst possible result 0 .

values of $Q^{\prime \prime}$ for groups with definite and possible gold standard diagnoses and for the cases in which the diagnosis in question was not made by any doctor. Note that $Q^{\prime \prime}$ is identical with the average overall probability in table 2 for definite doctor's diagnoses, but not for the other categories. The value of $\mathrm{Q}^{\prime \prime}$ for diagnoses not made by any doctor is high. Both models were therefore generally successful in excluding incorrect diagnoses - that is, assigning them a probability near 0 .

\section{Discussion}

Building decision support systems requires continuous evaluation of the diverse aspects of the system under consideration. Three phases of evaluation have been proposed: definition of the problem, validation of the knowledge, and clinical testing. ${ }^{15}$ In this report we present the first two phases for our diagnostic support system.

Here the first phase, definition of the problem, may be summarised as follows: our program is aimed primarily at the calculation of the probability of major rheumatic disease categories using Bayes's rule. More or less exact information about probabilities may be of help in diagnosing common rheumatological disorders. This may lead to several practical applications. Firstly, the program may suggest useful questions for anamnesis by comparing the evidential weights of findings. Secondly, it can provide a rational basis for the selection of diagnostic tests by calculating their posterior probability. From the data in table 1 it is clear that a large number of patients are referred with 'non-specific pain'. For most of these patients rheumatological treatment is of limited value. Therefore, thirdly, the number of referrals might be reduced if low probability for serious

Table 4 Performance by probability of the computer prediction

\begin{tabular}{|c|c|c|c|c|c|c|c|c|c|c|c|c|}
\hline \multirow[t]{2}{*}{ Prediction } & \multicolumn{6}{|c|}{ Classical formula (I) } & \multicolumn{6}{|c|}{ Modified formula (3) } \\
\hline & $\overline{n^{*}}$ & $P^{*}$ & $\operatorname{Exp}^{*}$ & Obs* & $S E^{*}$ & $Z^{*}$ & $n$ & $P$ & $\operatorname{Exp}$ & Obs & $S E$ & $Z$ \\
\hline $\begin{array}{l}0.95-1.0 \\
0.80-0.94 \\
0.60-0.79 \\
0.40-0.59 \\
0.20-0.39 \\
0.10-0.19 \\
0.05-0.09 \\
0.02-0.04 \\
0.00-0.01\end{array}$ & $\begin{array}{r}162 \\
128 \\
139 \\
148 \\
200 \\
255 \\
273 \\
393 \\
1152\end{array}$ & $\begin{array}{l}0.98 \\
0.88 \\
0.69 \\
0.49 \\
0.28 \\
0.14 \\
0.07 \\
0.03 \\
0.003\end{array}$ & $\begin{array}{r}159 \\
112 \\
96 \\
73 \\
57 \\
35 \\
18 \\
11 \\
4\end{array}$ & $\begin{array}{r}144 \\
109 \\
103 \\
82 \\
85 \\
61 \\
52 \\
42 \\
57\end{array}$ & $\begin{array}{l}1 \cdot 61 \\
3.71 \\
5 \cdot 46 \\
6 \cdot 08 \\
6 \cdot 38 \\
5 \cdot 50 \\
4 \cdot 13 \\
3 \cdot 24 \\
1 \cdot 89\end{array}$ & $\begin{array}{r}-9.58 \\
-0.89 \\
1.33 \\
1.47 \\
4.39 \\
4.70 \\
8.16 \\
9.62 \\
28.32\end{array}$ & $\begin{array}{r}218 \\
124 \\
114 \\
99 \\
156 \\
205 \\
297 \\
394 \\
1243\end{array}$ & $\begin{array}{l}0.98 \\
0 \cdot 88 \\
0 \cdot 71 \\
0 \cdot 49 \\
0 \cdot 28 \\
0 \cdot 14 \\
0 \cdot 07 \\
0 \cdot 03 \\
0.003\end{array}$ & $\begin{array}{r}215 \\
109 \\
80 \\
49 \\
44 \\
28 \\
20 \\
11 \\
4\end{array}$ & $\begin{array}{r}203 \\
104 \\
85 \\
60 \\
69 \\
63 \\
46 \\
43 \\
54\end{array}$ & $\begin{array}{l}1 \cdot 81 \\
3.62 \\
4 \cdot 87 \\
4 \cdot 97 \\
5 \cdot 61 \\
4.94 \\
4 \cdot 33 \\
3.28 \\
1 \cdot 89\end{array}$ & $\begin{array}{r}-6.47 \\
-1.41 \\
0.94 \\
2.30 \\
4.50 \\
7.03 \\
5.98 \\
9.73 \\
26.60\end{array}$ \\
\hline
\end{tabular}

${ }^{*} \mathrm{n}=$ number of predictions; $\mathrm{P}=$ average predicted probability; $\mathrm{Exp}=$ expected number of correct diagnoses ( $\mathrm{n} \times$ average probability); Obs $=$ observed number of cases in each category with a correct diagnosis; $S E=$ standard error of expected number $=\{\exp (1-\exp / n) !$
$Z=Z$ statistic derived by the formula: $Z=($ observed - expected $) /(\mathrm{SE})$. Values of $Z$ between 2 and -2 are optimal $($ see ref 12$)$. 
rheumatic disease could be readily established. An area of application common to all expert systems is the distribution of knowledge and postgraduate teaching in particular. ${ }^{16} \mathrm{~A}$ computer program for the diagnosis of common rheumatic complaints should prove to be of use to doctors who are unfamiliar with the field.

Bayesian likelihood estimates have been applied successfully to diagnostic problems. A well known example is the diagnosis of abdominal pain by DeDombal et al. ${ }^{17} 18 \mathrm{~A}$ limitation of statistical approaches to computer aided diagnosis is that they are not well suited to the diagnosis of rare diseases, as are expert systems using production rules or criteria tables, such as AI/RHEUM. ${ }^{4-6}$ A second limitation is to be found in the transferability of knowledge derived from a particular clinic to other medical situations, which may vary from general practice to university hospitals. ${ }^{14} 19$ This concerns both the prevalence of categories of rheumatic diseases and likelihood ratios of signs and symptoms. An advantage of the probabilistic approach is the quantitative representation of the likelihood of diagnoses. In particular, in a domain such as rheumatology where 'possible' or 'probable' diagnoses are common this may be more useful than a mere listing of one or more diagnoses.

The central question in the second phase of evaluation, validation of the knowledge in a decision support system, is whether its diagnoses are accurate. Measurement of the performance of computer predictions of diagnoses has been described extensively for systems with single correct diagnoses. ${ }^{20-22}$ The gold standard with which we compared the performance of the computer program included more than one diagnosis at multiple possible confidence levels for each case. There are thus a variety of measures available to evaluate the system's performance. We have concentrated on questions a prospective user may have: How accurate is the system at predicting or excluding a particular diagnosis? How often are diagnoses correct by rank in the order of presentation? Does a given probability correspond to a fraction of patients for whom the diagnosis in question is correct? These questions may be answered by referring to tables $2,3,4$ and the figure. The methods are relatively simple but provide adequate insight into the actual performance of the system. In particular, the metrics we have applied reflect accuracy of the predictions rather than numbers of correctly diagnosed cases. The results indicate little difference between the two variations of Bayes's theorem. Nevertheless, a higher level of performance is required before the program can enter a phase of clinical evaluation. This may be achieved by further modifications of the list of questions or the method of calculation (the model), or by using sources to derive likelihood ratios (the knowledge).

Regarding the model, for example, in calculating the probabilities there is some question whether these should be normalised so that their sum is 1 . Although it is natural to try to explain a set of findings by a single diagnosis, one third of our patients were given more than one. It may therefore be preferable to calculate the probability of each diagnosis independently. The diagnostic accuracy will presumably not deteriorate when the number of questions in the model is reduced. If dependence of findings would be avoided in a smaller list, the results might even improve. We explore this issue in subsequent studies in order to balance statistical and clinical preferences regarding the number of data items.

The knowledge might be refined in various ways. More sophisticated methods of deriving weights that would account for the interdependence of findings and statistical confidence of the sensitivity and specificity may lead to better results. Review of the weights by a rheumatologist would make it possible to incorporate widely accepted definitions, such as the age limit of 16 years for rheumatoid arthritis. We are currently assessing the effect of such changes in the method of calculation and the knowledge by means of the measures of performance described here. The transferability of the system may be evaluated by the same methods with data from other patient groups.

Our study illustrates the use of one form of automated knowledge acquisition. The knowledge used by the program is not obtained directly from human experts, as is the case in most rule based expert systems, but is derived from a set of patients data with gold standard diagnoses made by human experts. Thus, given sufficiently large databases, the methodology may be of use in refining classification criteria or even design truly diagnostic criteria that accurately reflect the likelihood of a disease or a particular diagnostic outcome. ${ }^{23} 24$

The use of Bayes's rule or variations of it in a computer program for the differential diagnoses of major rheumatic disease categories is practicable. Such a system may have numerous useful applications. The only proof of its usefulness, however, will be successful implementation in day to day patient care.

This work was supported by a grant from the Dutch League against Rheumatism. The authors are grateful to their colleague at the Jan van Breemen Institute, who filled out the dat collection forms.

1 Arnett F C, Edworthy S M, Bloch D A, et al. The American Rheumatism Association 1987 revised criteria for the class fication of rheumatoid arthritis. Arthritis Rheum 1988; 31 : 315-24.

2 Reggia J A, Tuhrim S. An overview of methods for computerassisted medical decision making. In: Reggia J A, Tuhrim S, eds. Computer-assisted medical decision making. Vol 1 . New eds. Computer-assisted medical

3 Waxman H S, Worley W E. Computer-assisted adult medical diagnosis: subject review and evaluation of a new microdiagnosis: subject review and evaluation of a new microcomputer

4 Kingsland L C, Lindberg D A B, Sharp G C. AI/Rheum. A consultant system for rheumatology. F Med Syst 1983; 7 $221-7$.

5 Kingsland L C. The evaluation of medical expert systems: experience with the $\mathrm{AI} / \mathrm{Rh}$ heum knowledge based consultan system in rheumatology. Proc Ninth Annual SCAMC. Washington, DC:IEEE Computer Society Press, 1985: 292-5.

6 Porter J F, Kingsland L C, Lindberg D A B, et al. The AI/RHEUM knowledge-based computer consultant system in rheumatology. Performance in the diagnosis of 59 connective tissue disease patients from Japan. Arthritis Rheum 1988; 31: 219-26.

7 Schewe S, Herzer P, Kruger K. Prospective application of an expert system for the medical history of joint pain. Klin Wochenschr 1990; 68: 466-71. 
8 Kolarz G, Adlassnig K P. Problems in establishing the medical expert systems CADIAG-1 and CADIAG-2 in rheumatology. F Med Syst 1986; 10: 395-405.

9 Horn W. Utilizing detailed anatomical knowledge for hypothesis formation and hypothesis testing in rheumatological decision support. Artificial Intelligence in Medicine 1991; 3: 21-39.

10 Bürer J H, Groot A, van de Laar M A F J, Olsthoorn P G, van Soesbergen $R$ M, van der Korst J K. Bacterial joint infections: a retropective study for the source of the infection. Ned Tijdschr Geneeskd 1989; 113: $1693-6$.

11 McNeil B J, Keeler E, Adelstein S J. Primer on certain elements of medical decision making. $N$ Engl $\mathcal{F}$ Med 1975; 293: 211-5.

12 Spiegelhalter D J. Statistical methodology for evaluating gastrointestinal symptoms. Clin Gastroenterol 1985; 14: gastrointest

13 Shapiro A R. The evaluation of clinical predictions: a method and initial application. $N$ Engl f Med 1977; 296: 1509-14.

14 Zagoria R J, Reggia J A. Transferability of medical decision support systems based on Bayesian classification. Med Decis Making 1983; 3: 501-9.

15 Wyatt J, Spiegelhalter D J. Evaluating medical expert systems: what to test and how? Med Inf (Lond) 1990; 15: 205-17.

16 Lindberg D A B, Sharp G C, Kay D R, et al. The expert consultant as teacher. MOBIUS 1983; 3: 30-6.

17 DeDombal F T, Leaper D J, Staniland J R, McCann A P,
Horrocks J C. Computer-aided diagnosis of acute abdominal pain. $B M \mathcal{J}$ 1972; ii: 9-13.

18 Adams I D, Chan M, Clifford P C, et al. Computer aided diagnosis of abdominal pain: a multicentre study. $B M \mathcal{F}$ 1986; 293: 800-4.

19 Lindberg G, Seensalu R, Nilsson L H, Forsell P, Kager L, Knill-Jones R P. Transferability of a computer system for medical history taking and decision support in dyspepsia. A comparison of indicants for peptic ulcer disease. Scand $f$ Gastroenterol 1986 (suppl 128): 190-6.

20 Habbema J D F, Hilden J, Bjerregaard B. The measurement of performance in probabilistic diagnosis (parts 1-3). of performance in probabilistic
Methods Inf Med 1978; 17: 217-46.

21 Habbema J D F, Hilden J, Bierregaard B. The measurement of performance in probabilistic diagnosis (parts 4 and 5). Methods Inf Med 1981; 20: 80-100.

22 Habbema J D F. Models for diagnosis and detection of combinations of diseases. In: DeDombal F T, Gremy F, eds. Decision making and medical care. Amsterdam: North Holland, 1976: 399-411.

23 Balint G P, Buchanan W W. Diagnosis of rheumatic disease: a plea for contemplation of the future. $B r \mathcal{Y}$ Rheumatol 1986; 25: $399-401$.

24 Cheeseman P, Kelly J, Self M, Stutz J, Taylor W, Freeman D. AutoClass: a Bayesian classification system. Proceedings of the Fifth International Conference on Machine Leaming, Ann Arbor: University of Michigan, 1988: 54-64. 